\title{
Comparison of k-NN, SVM, and NN in Pit Pattern Classification of Zoom-Endoscopic Colon Images using Co-Occurrence Histograms *
}

\author{
M. Häfner, A. Gangl, F. Wrba \\ Vienna Medical University, Austria \\ K. Thonhauser, H.-P. Schmidt \\ Carinthia Tech Institute, Klagenfurt, Austria
}

\author{
Ch. Kastinger, A. Uhl \\ Salzburg University, Austria \\ A. Vécsei \\ St. Anna Children's Hospital, Austria
}

\begin{abstract}
Co-occurrence histograms are used as features to classify magnifying endoscope imagery with $k-N N, S V M$, and $N N$ classifiers. In the $k-N N$ classification case these histograms may improve the classification accuracy of simple $1 D$ color histograms up to $10 \%$ in the 2 classes case and up to $5 \%$ in the 6 classes case. The classification results of SVM and NN classifiers have turned out to be noncompetitive and do not improve the classification result of $1 D$ color histograms.
\end{abstract}

\section{Introduction}

Colonoscopy is a medical procedure which makes it possible for a physician to evaluate the appearance of the inside of the colon. This is done by using a colonoscope. A colonoscope is a flexible, lighted instrument which enables physicians to view the colon from inside without any surgery involved. If the physician detects suspicious tissue he might also obatin a biopsy, which is a sample of suspicious tissue used for further examination under a microscope. Some colonoscopes are also able to take pictures and video sequences from inside the colon during the colonoscopy. This allows a physician to review the results from a colonoscopy to document the growth and spreading of an eventual tumorous lesion.

Another possibility arising from the ability of taking pictures from inside the colon is analysis of the images or video sequences with the assistance of computers. This allows computer assisted detection of tumorous lesions by analyzing video sequences or images where the latter one is the main focus of this work.

To get images which are as detailed as possible a special endoscope - a magnifying endoscope - can be used to create the set of images used throughout this thesis. A magnifying endoscope represents a significant advance in colonoscopic diagnosis as it provides images which are up to 150 -fold

$*$ The work described in this paper is partially supported by the Austrian Science Fund, project no. L366-N15. magnified. This magnification is possible through an individually adjustable lens. Images taken with this type of endoscope are very detailed as they uncover the fine surface structure of the mucosa as well as small lesions. To visually enhance the structure of the mucosa and therefore the structure of an eventual tumorous lesion, a common procedure is to spray indigo carmine or metyhlen blue onto the mucosa.

In this work we employ co-occurrence histograms and compare different types of classification techniques for an automated classification of visual data aquired by a magnifying colonoscope corresponding to different types of lesions. In Section 2, we review the classification of pit patterns of the colonic mucosa. Section 3 gives an overview of feature extraction and classification procedures employed. Experimental results and configuration details of the system proposed in this work are presented and discussed in Section 4. Section 5 concludes this work.

\section{Pit Pattern Classification}

Polyps of the colon are a frequent finding and are usually divided into metaplastic, adenomatous, and malignant. As resection of all polyps is time-consuming, it is imperative that those polyps which warrant endoscopic resection can be distinguished: polypectomy of metaplastic lesions is unnecessary and removal of invasive cancer may be hazardous. For these reasons, assessing the nature of lesions at the time of colonoscopy is important. Diagnosis of tumorous lesions by endoscopy is always based on some sort of staging, which is a method used to evaluate the progress of cancer in a patient and to see to what extent a tumorous lesion has spread to other parts of the body. Staging is also very important for a physician to choose the right treatment of the colorectal cancer according to the respective stage. A recent classification system, based on so-called pit patterns of the colonic mucosa, was originally reported by Kudo et al. $[9,10]$. As described in Table 1 this classification differentiates between five main types according to the mucosal surface of the colon. The higher the type number the higher is the risk of a lesion to be malignant: It has been suggested that type I and II pattern are characteristic of non-neoplastic lesions, type III and IV are found on adenomatous polyps, 
and type $\mathrm{V}$ are strongly suggestive of invasive carcinoma. While lesions of type I and II are benign, representing the normal mucosa or hyperplastic tissue, and in fact are nontumorous, lesions of type III to V in contrast represent lesions which are malignant. Lesions of type I and II can be grouped into non-neoplastic lesions, while lesions of type III to $\mathrm{V}$ can be grouped into neoplastic lesions. Thus a coarser grouping of lesions into two instead of six classes is also possible.

\begin{tabular}{|cl|}
\hline Pit & Characteristics \\
\hline I & roundish pits which designate a normal mucosa \\
II & stellar or papillary pits \\
III S & $\begin{array}{l}\text { small roundish or tubular pits, which are smaller } \\
\text { than the pits of type I }\end{array}$ \\
III L & $\begin{array}{l}\text { roundish or tubular pits, which are larger than } \\
\text { the pits of type I }\end{array}$ \\
IV & branch-like or gyrus-like pits \\
V & non-structured pits \\
\hline
\end{tabular}

\section{Table 1. Characteristics of different pit pattern types.}

Using a magnifying colonoscope together with indigo carmine dye spraying, the mucosal crypt pattern on the surface of colonic lesions can be observed [10]. Several studies found a good correlation between the mucosal pit pattern and the histological findings, where especially techniques using magnifying colonoscopes led to excellent results $[7,3]$.

Although at a first glance this classification scheme seems to be straightforward and easy to be applied, it needs some experience and exercising to achieve fairly good results. Correct diagnosis very much relies on the experience of the endoscopist as the interpretation of pit patterns may be challenging [6]. To show this, figure 1 contains images out of the training image set used in this study.

Additionally, inter-observer variability of magnification chromoendoscopy has been described at least for Barrett's esophagus [13]. This inter-observer variability may to a lesser degree be also present in the interpretation of pit patterns of colonic lesions. We therefore want to develop an algorithm for computer based pit pattern analysis in order to get objective and reproduceable results.

\section{Computer Assisted Classification of Endo- scopic Images}

If a computer program has to discriminate between different classes of images some sort of classification algorithm has to be applied to the training data during the training phase. During the subsequent classification of an unknown image, the formerly trained classification algorithm is applied to the new, unknown image and tries to classify it correctly. Thus the classification process mainly consist of two parts: the extraction of relevant features from images and the classification based on these features.

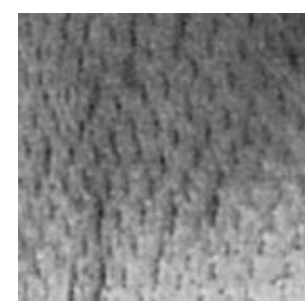

(a) Pit pattern I

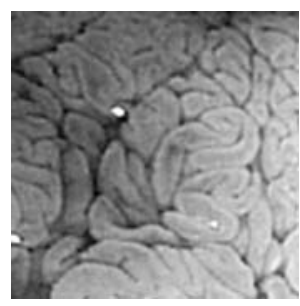

(c) Pit pattern IV
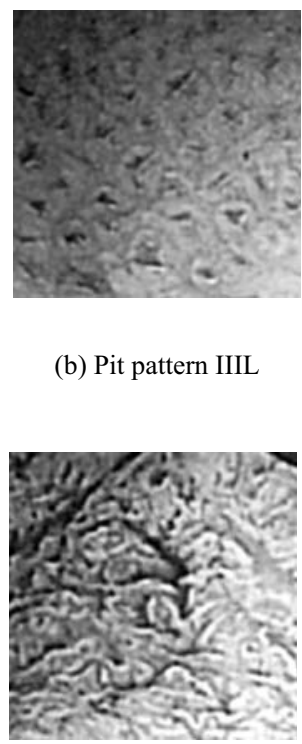

(d) Pit pattern V (b) Pit pattern IIIL

Figure 1. Images showing the different types of pit pattern.

Various techniques have been applied for computer assisted classification of endoscopic images, in many cases either spatial domain features (e.g. [15]) or transform domain features (e.g. like wavelet-based ones [8]) are fed into neural net based classifiers.

\subsection{Feature Extraction: Co-occurrence His- tograms}

Histograms have been widely used in classification [16], image retrieval [14], and object recognition [2] applications. One of the main reasons are the histograms' robustness to translation and rotation, and position invariance in general.

In recent work [4] we have found histogram-based techniques to be surprisingly well suited for our classification problem. This is quite surprising, since pit patterns seem to be more localized phenomena characterized by their respective shapes - however, the corresponding results still leave room for improvements. Here, we employ co-occurrence histograms as features to be fed into subsequent classification techniques.

The co-occurrence histogram $(\mathrm{CH})$ [2] counts of the number of pairs of pixels exhibiting specific colour values that occur at certain separation distances in image space. Therefore, the $\mathrm{CH}$ adds geometric information to the classical color histogram, which abstracts away all geometry. By adjusting the distances over which we check cooccurrences, we can adjust the sensitivity of the algorithm. We use horizontal and diagonal separation up to a Manhattan distance of 20 pixels. The $\mathrm{CH}$ is constructed based on different colour channels. This results in a 2-dimensional histogram where the colour values of the two pixels under 
investigation represent the two dimensions.

Fig. 2(a) shows how the histogram is accumulated for two specific pixels at the required spatial distance. Fig. 2(b) shows a simple example of a co-occurrence histogram of the data.

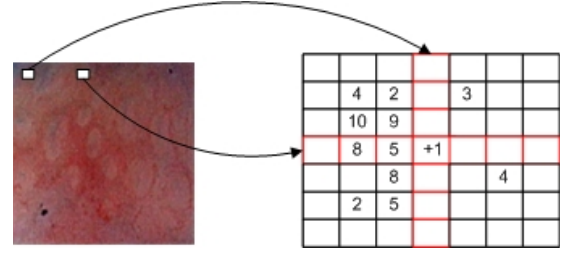

(a) construction principle

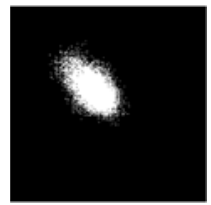

(b) example
Figure 2. Co-occurrence histograms.

\subsection{Classification}

One of the simplest classification techniques is the $k$ Nearest Neighbour (k-NN) classifier. Classification of an input feature vector $\vec{x}$ is done by determining the $k$ closest training vectors according to a suitable distance metric. The vector $\vec{x}$ is then assigned to that class to which the majority of those $k$ nearest neighbours belong to.

One of the most popular ways to determine histogram distance is to employ histogram intersection [11]. Given a pair of histograms, $H(I)$ and $H\left(I^{\prime}\right)$, of images $I$ and $I^{\prime}$, respectively, each containing $n$ bins, the histogram intersection of the normalized histograms is defined as follows:

$$
H(I) \cap H\left(I^{\prime}\right)=\sum_{j=1}^{n} \min \left(H_{j}(I), H_{j}\left(I^{\prime}\right)\right)
$$

For two images, the larger the value of the histogram intersection, the more similar the image pair is deemed to be. Therefore, histogram intersection may be immediately used for k-NN classification and has turned out to give good results on simple histograms and wavelet-based features derived from zoom-endoscopic images in earlier work $[5,4]$.

Artificial neural networks (ANNs) have already been applied for endoscopic image classification before in various flavours: for example we mention Multilayer Feed Forward Neural Networks (MFNN) [12] and Backpropagation Neural Networks (BPNN) [15].

Finally, we have also already used Support Vector Machines (SVM) to classify wavelet-based feature vectors derived from zoom-endoscopic images in previous work [5].

\section{Experimental Study}

\subsection{Settings and Methods}

In our experiments we use 484 images aquired in 2005 - 2006 at the Department of Gastroenterology and Hepatology using a zoom-colonoscope (Olympus Evis Exera CF-Q160ZI/L) with a magnification factor set to 150 . Lesions found during colonoscopy have been examined after application of dye-spraying with indigocarmine. Biopsies or mucosal resection have been performed in order to get a histopathological diagnosis. Biopsies have been taken from type I, II, and type V lesions, as those lesions need not to be removed or cannot be removed endoscopically. Type III and IV lesions have been removed endoscopically. Out of all aquired images, histopathological classification resulted in 198 non-neoplastic and 286 neoplastic cases. The detailed classification results - which are used as ground truth for our experiments - are shown in Table 2.

\begin{tabular}{|l||c|c|c|c|c|c|}
\hline Pit Type & I & II & IIIS & IIIL & IV & V \\
\hline Images & 126 & 72 & 18 & 62 & 146 & 60 \\
\hline
\end{tabular}

\section{Table 2. Number of images per class used in experiments.}

The acquired colour images are investigated with separated colour channels in three different colour models: RGB (as being provided initially), $\mathrm{YCrCb}$, and $\mathrm{HSV}$. Since the available image set is still rather small for a classification application, we cannot afford separate training and test sets. 483 out of 484 images are used as training set, the remaining image is then classified. This process ("leave-one-out" technique) is repeated for each single image.

Besides k-NN classification, we employ SVM and Neural Network-based (NN) classification. Contrasting to k-NN classification, the data dimensionality of the co-occurrance histogram is much too high to be fed directly into these classifiers. Fig. 3 shows the stages to handle this issue. First, the resolution of the histogram is reduced by a factor of 2 in each dimension. Subsequently, the vectorized data is subjected to a PCA and the data is approximated using a limited number of the most significant eigenvectors. The amount of vectors employed has a significant impact on the classification result as we shall see (Figs. 8 and 9).

We use a 3-layer feed forward neural network with nonlinear transfer functions (logarithmic-sigmoid) in the hidden and output layer. The resilient back propagation algorithm is employed. The number of input neurons has been set equal to size of the feature vector obtained from the the PCA feature selection and the number of output neurons is fixed to the number of classes. To ensure that the backpropagation algorithm converges to the global minimum of error instead of a less optimal minimum the initial layer weights have to be optimized. Therefore a high number of randomly created configurations have been evaluated based on equal input data with regard to the average classification accuracy, to find a setting near the global minimum. The most promising initial layer weights have been stored in a file and used for all later investigations.

Concerning SVM classification, the LIBSVM library is used [1]. We conduct a naive grid search to find the best parameter choices for our classification purpose. The applied SVM classifier is a $v$-soft margin version with one-againstone approach and a RBF-Kernel. For optimal classification performance both the Kernel parameter $\gamma$ and the soft margin parameter $0<v<1$ have been optimized with regard 

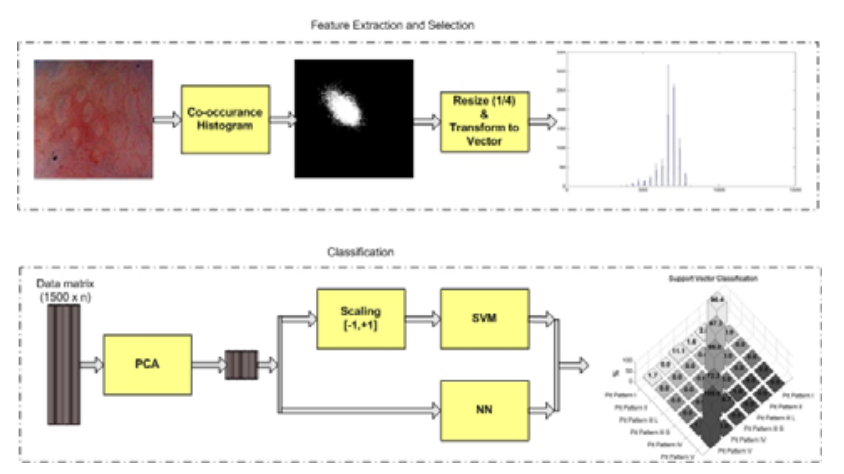

Figure 3. Structure of SVM and NN based classification.

to the average classification accuracy. The best results have been achieved for $\gamma=0.1$ and $v=0.5$ (compare Fig. 7). To enable classification the input data have been normalized to values between $[+1,-1]$.

\subsection{Experimental Results}

Figs. 4(a) and 4(b) show the results of applying k$\mathrm{NN}$ classification to simple 1D colour histograms of single colour channels in the two classes case (this result just applies the technique described in [4] to the larger data set). The best result in terms of percentage of correctly classified images is shown for each colour model RGB, HSV, and $\mathrm{YCrCb}$. The RGB model consistently delivers the worst result. For the two-class case, $\mathrm{HSV}$ and $\mathrm{YCrCb}$ are close whereas in the 6 class case $\mathrm{YCrCb}$ is always better. Small $\mathrm{k}$-values tend to give better results at least for HSV and $\mathrm{YCrCb}$. The best results found are $80 \%$ in the 2 class case $(\mathrm{k}=1$ for $\mathrm{H}(\mathrm{SV})$ and $(\mathrm{Y}) \mathrm{Cr}(\mathrm{Cb}))$ and $70 \%$ in the 6 class case $(\mathrm{k}=1$ for $(\mathrm{Y}) \mathrm{Cr}(\mathrm{Cb}))$.

In the following we want to investigate whether $\mathrm{CH}$ may improve on these results. We begin with discussing k-NN classification results. It turns out that $\mathrm{CH}$ constructed from colour channels do not improve on the results of simple colour histograms in the cases of the RGB and HSV colour models. The results for the third colour model $\mathrm{YCrCb}$ are shown in Figs. 5(a) and 5(b). Here the best results are seen for the Y channel and again for $\mathrm{k}=1$ with $90 \%$ for the 2 classes case and $75 \%$ for the 6 classes case. Interestingly, in the 1D histogram case the best results have been achieved for the Cr channel (compare Figs. 4(a) and 4(b)), whereas for $\mathrm{CH}$ the $\mathrm{Y}$ channel is clearly superior.

So far, the results have been achieved using a vertical and horizontal displacement of 1 pixel each in the construction of the $\mathrm{CH}$ (i.e. two adjacent pixel in a 8-neighbourhood are considered). In the following, we extend this spatial relation up to a Manhattan distance of 20 pixel, always using an equal amount of horizontal and vertical displacement). Fig. 6(a) shows the results for the G channel. The best results found are for a Manhatten distance of $12-16$ (shifts of $6-8$ in both directions) for both the 2 classes and 6 classes case (the latter is not shown). Overall, the differences are small. Fig. 6(b) reveals that for the Y channel the spatial

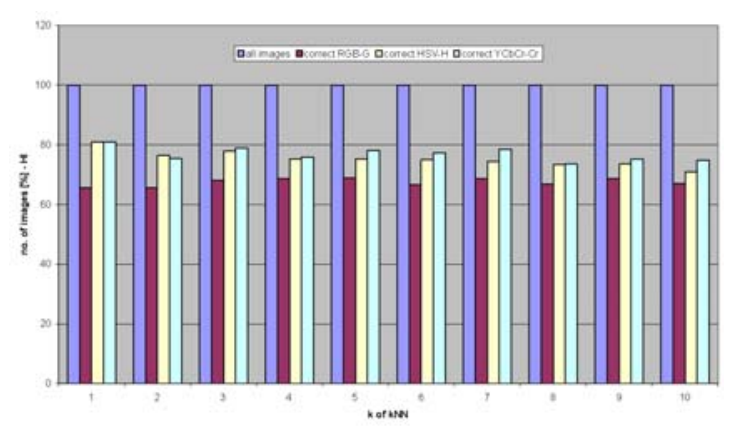

(a) 2 classes

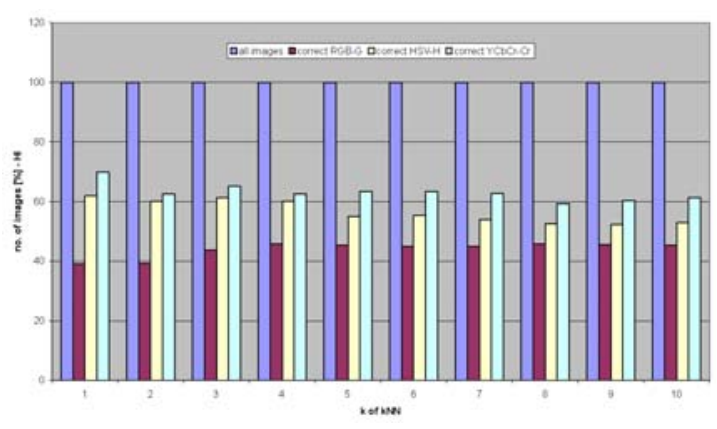

(b) 6 classes

Figure 4. Percentage of correctly classified images for 1D colour histograms.

distance considered does not matter at all. This is quite surprising since the $\mathrm{Y}$ channel $\mathrm{CH}$ is the best setting encountered. This result indicates that the extent of the localization aspect brought into the scheme by $\mathrm{CH}$ is not important for the result achieved.

In the following, we discuss the results obtained by SVM and NN classification, respectively. Fig. 7 shows the importance of optimizing the Kernel parameter $\gamma$ and the soft margin parameter $0<v<1$ in the SVM case. We use the 70 most important eigenvectors from the PCA and a co-occurrence distance of 4 horizontal pixels. The classification accuracy for the $\mathrm{Y}$ channel varies significantly in the ranges considered. Based on a large number of experiments, these parameters have been fixed at $\gamma=0.1$ and $v=0.5$.

Fig. 8 shows the influence of the number of eigenvectors used in the PCA-based approximation on the classification results for the NN and SVM approaches, respectively. It turns out that a low amount leads to inferior results (as it is expected), but also an amout exceeding a certain threshold leads to detoriated results. Based on these experiments, we set the number of eigenvectors employed to 70. The overall classification accuracy achieved is $78 \%$ with SVM and $72 \%$ with NN classification in the 2 classes case. Note that this is slightly below the values of $1 \mathrm{D}$ histograms and clearly inferior to the $\mathrm{CH}$ k-NN results. 


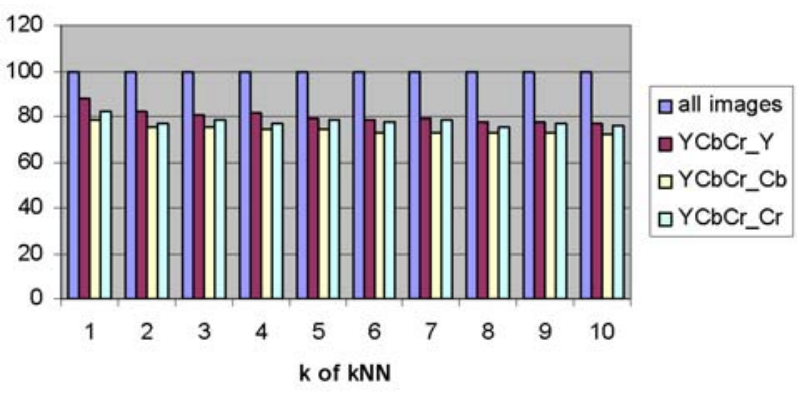

(a) 2 classes

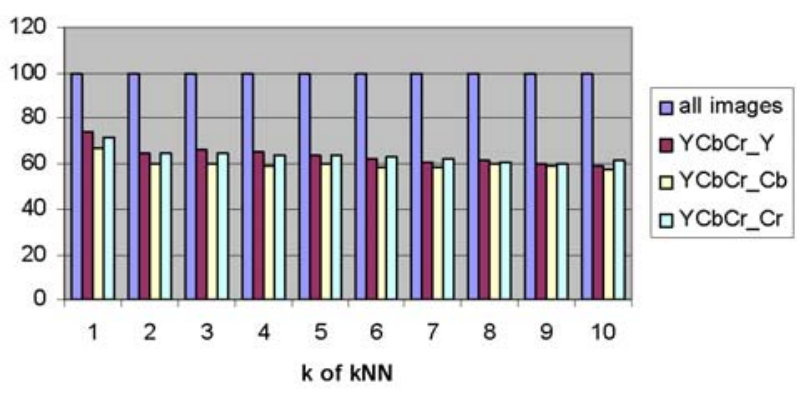

(b) 6 classes

Figure 5. Percentage of correctly classified images for co-occurrence histograms in the YCrCb colourspace.

In Fig. 9 we consider the 6 classes case. The best classification rates of $52 \%$ for SVM and $41 \%$ for NN clearly show that these types of classification schemes severly suffer from a low amount of training data available.

Finally, we investigate the impact of the co-occurrence distance by increasing the horizontal distance up to 20 pixels. We find that there is a point of optimal operation at a distance $7-9$, we result in a recognition accuracy of $80 \%$ for SVM and $75 \%$ for $\mathrm{NN}$ in the 2 classes case. However, this is still clearly inferior to the k-NN classifier results and does not improve the results of the 1D histograms.

\section{Conclusions}

In the classification of zoom-endoscopic colon imagery we have found that co-ocurrence histograms may improve the classification accuracy of simple 1D color histograms up to $10 \%$ in the 2 classes case and up to $5 \%$ in the 6 classes case. However, this is only true for $\mathrm{k}=1$ in the $\mathrm{k}$ NN classifier and the Y colour channel. The classification results of SVM and NN classifiers have turned out to be non-competitive, probably due to the massive dimensionality reduction required by these schemes. It is interesting to note that the spatial characteristic of the co-occurrance relation used for constructing the histograms has only minor influence on the classification results (especially for the top ranked method).

In future work we will concentrate on clarifying the rea-

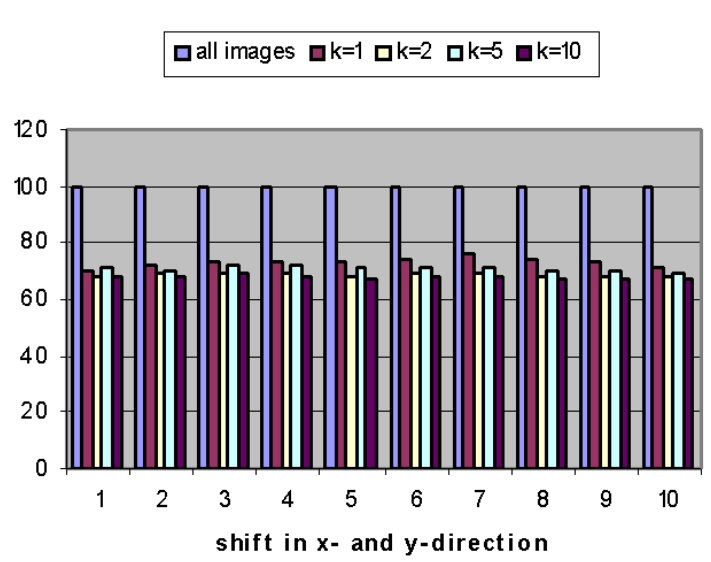

(a) G channel (RGB)

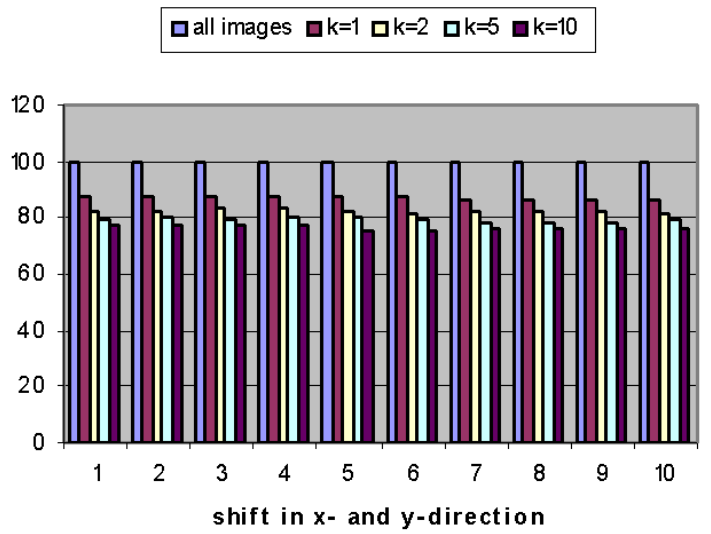

(b) Y Channel (YCrCb)

Figure 6. Percentage of correctly classified images for co-occurrence histograms, depending on spatial distance ( 2 classes case).

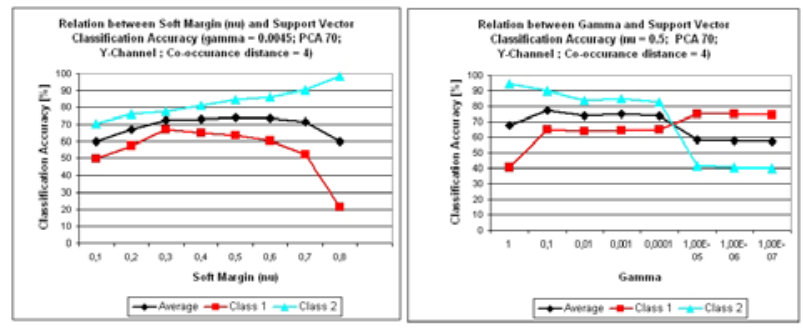

Figure 7. Parameter dependency of SVM classification results.

son for the inferior performance of SVM and NN classification. In the current setup, it is not entirely clear whether the poor SVM/NN performance is due to the classification process itself or due to loss of information in preprocessing with PCA. Therefore, on the one hand we will use the $\mathrm{CH}$ in $\mathrm{k}-\mathrm{NN}$ classification in the same way as with SVM and NN (i.e. applying subsampling and a PCA), on the other 


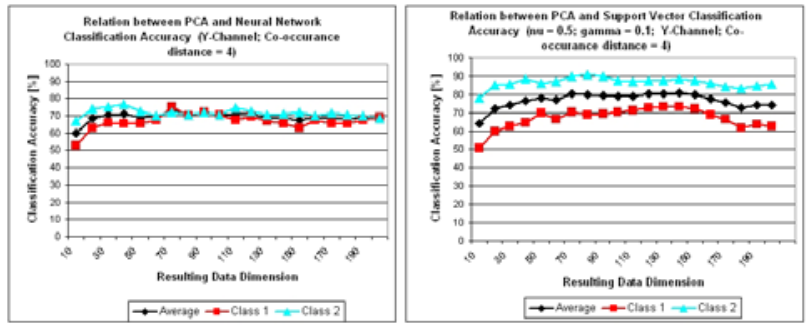

Figure 8. Impact of dimensionality reduction on classifcation results ( 2 classes case).

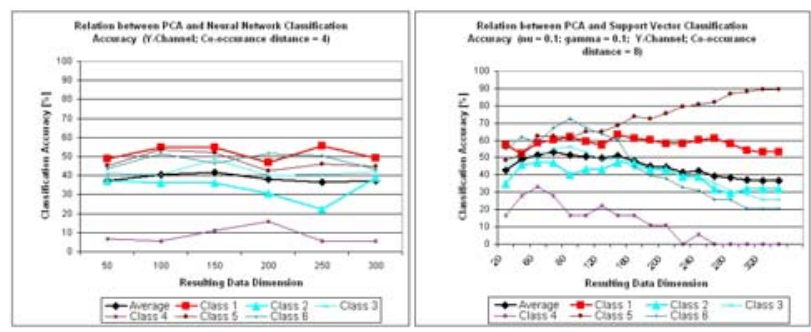

Figure 9. Impact of dimensionality reduction on classifcation results (6 classes case).

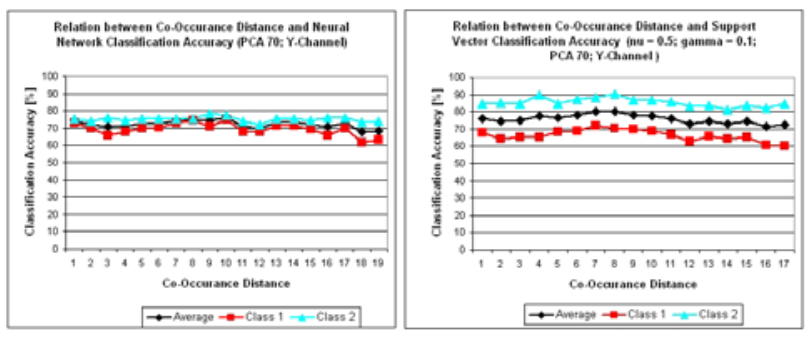

Figure 10. Impact of spatial characteristic of co-occurrance relation on classification results.

hand we will use the histogram intersection as a kernel for measuring similarity of histograms. Finally, instead of applying dimensionality reduction by PCA, we will consider using standard texture measures on the $\mathrm{CH}$ (such as histogram moments or entropy) before classification with k$\mathrm{NN}, \mathrm{SVM}$, and NN.

\section{References}

[1] C.-C. Chang and C.-J. Lin, LIBSVM: A Library for Support Vector Machines, 2001, Software available at http://www.csie.ntu.edu.tw/ cjlin/libsvm.

[2] P. Chang and J. Krumm, "Object recognition with color cooccurrence histogram", In Proceedings of CVPR '99, 1999.

[3] K.-I. Fu et al., "Chromoendoscopy using indigo carmine dye spraying with magnifying observation is the most reliable method for differential diagnosis between non-neoplastic and neoplastic colorectal lesions: a prospective study", Endoscopy, 36(12), 2004, pp. 1089-1093.
[4] M. Häfner, C. Kendlbacher, W. Mann, W. Taferl, F. Wrba, A. Gangl, A. Vécsei, and A. Uhl, "Pit pattern classification of zoom-endoscopic colon images using histogram techniques", In J. R. Sveinsson, editor, Proceedings of the 7th Nordic Signal Processing Symposium (NORSIG 2006), pp. 58-61, Reykavik, Iceland, 2006. IEEE.

[5] M. Häfner, M. Liedlgruber, F. Wrba, A. Gangl, A. Vécsei, and A. Uhl, "Pit pattern classification of zoom-endoscopic colon images using wavelet texture features", In W. Sandham, D. Hamilton, and C. James, editors, Proceedings of the International Conference on Advances in Medical Signal and Image Processing (MEDSIP 2006), Glasgow, Scotland, UK, 2006. paper no. 0038.

[6] D. Hurlstone, "High-resolution magnification chromoendoscopy: Common problems encountered in "pit pattern" interpretation and correct classification of flat colorectal lesions", American Journal of Gastroenterology, 97, 2002, pp. 1069-1070.

[7] D. Hurlstone et al., "Efficiacy of high magnification chromoscopic colonoscopy for the diagnosis of neoplasia in flat and depressed lesions of the colorectum: a prospective analysis", Gut, 53, 2004, pp. 284-290.

[8] S. A. Karkanis, "Computer-aided tumor detection in endoscopic video using color wavelet features", IEEE Transactions on Information Technology in Biomedicine, 7(3), September 2003, pp. 141-152.

[9] S.-E. Kudo et al., "Colorectal tumorous and pit pattern", Journal of Clinical Pathology, 47, 1994, pp. 880-885.

[10] S.-E. Kudo et al., "Diagnosis of colorectal tumorous lesions by magnifying endoscopy", Gastrointestinal Endoscopy, 44(1), July 1996, pp. 8-14.

[11] S. Lee, J. Xin, and S. Westland, "Evaluation of image similarity by histogram intersection", COLOR Research and Application, 30(4), 2005, pp. 265-274.

[12] D. Maroulis, D. Iakovidis, S. Karkanis, and D. Karras, "CoLD: a versatile detection system for colorectal lesions in endoscopy video-frames", In Computer Methods Programs Biomed., volume 70, pp. 151-166, February 2003.

[13] A. Meining et al., "Inter- and intra-observer variability of magnification chromoendoscopy for detecting specialized intestinal metaplasis at the gastroesophageal junction", Endoscopy, 36, 2004, pp. 160-164.

[14] A. Smeulders et al., "Content-based image retrieval at the end of the early years", IEEE Transactions on Pattern Analysis and Machine Intelligence, 22(12), 2000, pp. 1349-1380.

[15] M. P. Tjoa and S. M. Krishman, "Feature extraction for the analysis of colon status from the endoscopic images", BioMedical Enineering OnLine, April 2003.

[16] K. Valkealahati and E. Oja, "Reduced multidimensional co-occurrence histograms in texture classification", IEEE Transactions on Pattern Analysis and Machine Intelligence, 20(1), 1998, pp. 90-94. 